\title{
Phytochemical screening of Lycopersicum esculentum Mill. treated with seaweed liquid fertilizer
}

\author{
M. Niranjanadevi, K. Sivakumar* \\ Division of Algal biotechnology, Department of Botany, Annamalai University, Annamalai Nagar, India
}

\begin{abstract}
Premise of the study: The objective of the work is to manitor the phytochemicals present in methanolic extract of Lycopersicum esculentum after treatment with different concentrations of seaweed liquid fertilizer (SLF). Methods: In this study SLF treated L.esculentum were characterized through gas chromatography and mass spectroscopy to identify the phytochemicals constituents and functional groups of the compounds. Results: Preliminary phytochemical analysis revealed the presence of alkaloids, phenols, carbohydrates, saponins, glycosides, flavonoids, terpinoids. Among the thirty nine phytochemical constituents octa decanoic acid, stigmasterol, hexa decanoic acid and Vitamin E are relatively present in higher yield. The active phytochemical compounds and their constituents were identified with gas chromatography and mass spectrometry. The retention time, percentage of area, molecular weight and chemical formula of phytochemical compounds were determined with help of NIST08 and WILEY8 libraries. The functional groups identified from the spectrum techniques are alcohol, aldehyde, iso cyanides, alkyl compound and chloro compounds. Accordingly hexadecanic acid, beta-amyrin, gamma sisterol, octodecanic acid, phytol, stigmasterol, vitamin E, lupeol were derived from SLF treated i.e. compared to control plants. Conclusion: The results of this study offer a platform of using SLF treated L.esculentum has an alternative source for various biological studies and it can be used as functional and pharmaceutical purposes.
\end{abstract}

Keywords: SLF, Lycopersicum esculentum, Phytochemical, GC-MS and FTIR.

Article Info:

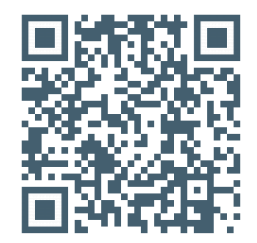

Cite this article as:

Niranjanadevi M, Sivakumar K, Phytochemical screening of Lycopersicum esculentum Mill. treated with seaweed liquid fertilizer, Journal of Drug Delivery and Therapeutics. 2018; 8(6-s):38-43

DOI: http://dx.doi.org/10.22270/jddt.v8i6-s.2195

*Address for Correspondence:

K. Sivakumar, Division of Algal biotechnology, Department of Botany, Annamalai university, Annamalai Nagar, India

\section{INTRODUCTION}

The tomato (Solanum lycopersicum, syn. Lycopersicon lycopersicum \& Lycopersicum esculentum) is an herbaceous, usually sprawling plant in the Solanaceae or nightshade family that is typically cultivated for the purpose of harvesting its fruit for human consumption. Tomato is rich in phenolic compounds (flavonoids and phenolic acids), phytoalexins, protease inhibitors, glycol-alkaloids and carotenoids, especially lycopene and b-carotene ${ }^{1}$ Rajkumar et al., 2004. The entire flora having the phytoconstituents and it's openly accepted. That we consume a wide variety of fruits and vegetables in order to increase maximum benefit from the nutrients and phytochemicals. Rather, consumption of phytochemicals should be from nutritive sources complements or drugs. These can only provide a few of the thousands of phytochemicals available to us and are thus less effective than a serving of fruits and vegetables $^{2}$ Harbone et al., 1973. Nowadays, secondary plant metabolites (phytochemicals), previously with unknown pharmacological activities, have been mostly investigated as a source of pharmaceutical agents ${ }^{3}$ Vandana Mathur et al., 2007.

Seaweed fertilizers used for sustained crop improvement and their potential uses for all over the decades. Seaweeds containing good effective nutritious and stimulate faster seedlings and increase yield and resistant ability of many crops. Liquid fertilizers derived from natural resources like seaweeds are found to be viable adaptations to fertilizing input for agricultural crops due to its high level of organic matter, micro and macro elements, vitamins, fatty acids also rich in growth promotors ${ }^{4}$ Crouch et al., 1993. Efficacy of seaweed extract to promote growth and yield of tomato plants and to improve lycopene and vitamin $C$ content of fruits, by different concentrations with aqueous extracts of Sargassum johnstonii ${ }^{5}$ (Kumari et al., 2011).

Identification of individual phytoconstituents such as terpenes, terpenoids in leaf and fruit methanol extracts requires the use of some techniques. GC-MS and FT-IR are powerful tools in pharmacological research intension for the identification and determination of chemical 
compounds and this technique has been previously applied successfully for the analysis of terpeniods. Especially mono and sesquiterpenes in various plant extracts. Identification of the bio molecules found in an extracts by comparing their relative rention times / indices and their mass spectra. In that way estimated the chemical constituents are used in folk medicine for variety of diseases including infectious conditions. In this present study an attempt to make in phytochemical screening of L.esculentum treated with seaweed liquid fertilizer compare with untreated plants.

\section{MATERIALS AND METHODS}

\section{Seaweed collection}

The marine brown seaweed Sargassum wightii was

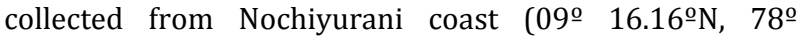
$02.43^{\circ} \mathrm{E}$ ) is located near madapam coast in the Gulf of mannar. The seaweeds were handpicked and washed thoroughly with seawater to remove all the unwanted sand particles.

\section{Seaweed liquid fertilizer preparation}

The cleaned and washed seaweeds were shade dried for ten days. After the dried material was taken grounded with the help of mixi grinder (Preethi ECO chef) the powdered seaweed samples were stored in the airtight container for the future use. $500 \mathrm{~g}$ of seaweed powder added to $5 \mathrm{~L}$ of water and heated for 45 mintutes at $60^{\circ} \mathrm{C}$ in plugged conical flask. After cooling the contents were filtered through four musclin cloths layers. The filtrate was centrifuged the supernatant collected was used as concentrated SLF. From the supernatant different concentrations (control, 10\%, $25 \%, 50 \%$ and $75 \%$ ) of SLF were prepared using distilled water.

\section{Experimental site}

The certified seeds of Lycopersicum esculentum PKM1 variety were procured from Agricultural research station in palur, Cuddalore (Dist) Tamil Nadu. The Tomato seeds were surface sterilized by 0.1 mercuric chloride and sown in earthenware pot in (10 X10 feet). The sterilized seeds were soked in each concentrations of SLF for $5 \mathrm{hrs}$ while control was maintained by soaking the seed, in a beaker containing equal level of distilled water. All the pot studies were done in the Botanical garden, Department of Botany, Annamalai University, Tamil Nadu.

\section{Chemicals}

The chemicals were obtained from Himedia, Mumbai, India and the solvents used were of analytical grade.

\section{Equipment}

Equipment's used in the experiment include GC-MS and FTIR. GC-MS was used for the comparison of samples and FTIR was used for identification of functional groups presented in the plant SLF (seaweed liquid fertilizer) treated tomato leaf.

\section{Preliminary phytochemical analysis}

Shade dried and powdered plant material was successively extracted with petroleum ether, chloroform, ethyl acetate and methanol with gentle stirring for 72 hrs separately. The extracts were filtered with whatman No.1 filter paper, and using vacuum distillation ${ }^{6}$ (Ncube et al., 2008). The preliminary phytochemical constituents were analysed qualitatively by using standard method was recorded in Table 1.

\section{GC-MS analysis}

Ten gram of powdered sample is extracted with $30 \mathrm{ml}$ methanol overnight and filtered in ashes filter paper with sodium sulphate (2) $g$ and the extract is concentrated to 1 $\mathrm{ml}$ by bubbling nitrogen into the solution. The clarus 500 GC used in the analysis employed a column packed with Elite -1 (100\% Dimethyl poly siloxane, $3 \mathrm{~nm}$ x $0.25 \mathrm{~nm}$ ID $\mathrm{x} 1 \mathrm{um} \mathrm{df}$ ) and the components were separated using helium $(1 \mathrm{~m} / \mathrm{min})$ as the carrier gas. The 2 ul sample extract injected into the instrument was detected by the Turbo mass gold mass detector (perkin Elmer) with the aid of the Turbo mass 5.1 software. During the $36^{\text {th }}$ minute GC extraction process, the over was maintained at a temperature was set at $25 \mathrm{C}$ (Mass analyser). The different parameters involved in the operation of Clarus $500 \mathrm{MS}$, were also standardized (inlet line temperature: $200 \mathrm{C}$; Source temperature $200 \mathrm{C}$; Electron energy $70 \mathrm{Ev}$; Mass scan $(\mathrm{m} / 2)$ 45-450). The MS detection was completed in 36 min. the relative percentage of each component was calculated by comparing its average peak to the total areas. The detection employed the NIST (National Institute of Standards and Technology) Version 2.0 year 2018 Library. The compound prediction is based on Dr. Duke's phytochemical and ethano botanical data analyses by Dr. Jim Duke of the agricultural Research Science/USDA.

\section{FT-IR Analysis}

Some of the fractions will be absorbed when infrared light passes through a sample of an organic compound; however, some frequencies will be transferred through the sample without any absorption occurring. Infrared absorption is related to the vibrational changes that happen inside a molecule when it is exposed to infrared radiation. Therefore, infrared spectroscopy can essentially be described as a vibrational spectroscopy. Different bonds (C-C, C=C, C_C, C-O, C=O, O-H, and $\mathrm{N}-\mathrm{H}$ ) have altered vibrational frequencies. If these kinds of chemical bonds are present in an organic molecule, they can be identified by detecting the characteristic frequency absorption band in the infrared spectrum Urban et al., 2006. Fourier Transform Infrared Spectroscopy (FTIR) is a highresolution analytical tool to identify the chemical components and revealed the structural compounds. FTIR offers a rapid and non destructive investigation to fingerprint herbal extracts or powders. The FT-IR analysis was completed using perkin Elmer Spectrum Version 10.03.09 system, which was used to identify the functional groups of the compound. A small amount of compound was placed directly on the zinc solenoid piece and constant pressure. Data of infrared absorbent, collected over the wave number ranged from $4000 \mathrm{~cm}^{-1}$ to $400 \mathrm{~cm}^{-1}$ using spectra software. Samples were run in triplicate and all of them were undertaken within a day period.

\section{RESULTS AND DISCUSSION}

Preliminary screening of phytochemical analysis of SLF treated L. esculentum exhibited phytochemical constituents were compared with the control or untreated plants. The PKM1 variety tomato seeds were sown in soil and SLF were added to soil bed in five different concentrations separately (10\%, 25\%, 50\%, 75\% and control). The SLF treated plants exhibited maximize growth and yield of $10 \%$ SLF among the various concentration as well as control. Further, the leaf of $10 \%$ SLF treated L. esculentum have subjected to phytochemical analysis its reveals that the presence of 39 Phytochemical constituents Hexadecanic acid, beta-amyrin, octadeconic acid, lupeol, vitamin E and Stigmasterol, majorly presented components from methanolic leaf 
extract (Table-3) and the following control leaf extract contain 28 phytochemical (Table-4). The spectra generated from study revealed that SLF of $S$. wightii could be used low concentration $10 \%$ of SLF treated plants phytochemicals components were identified. GC-MS analysis is a common confirmation test for determination of phytochemical constituents. GC-MS analyses separate all of the components in a sample and provide a representative spectral output. Each component ideally produces a specific spectral peak that may be recorded on a paper chart or electronically. The relation time can help to differentiate between some compounds. The sizes of the peaks are proportional to the quantity of the corresponding substances in the specimens analysed.

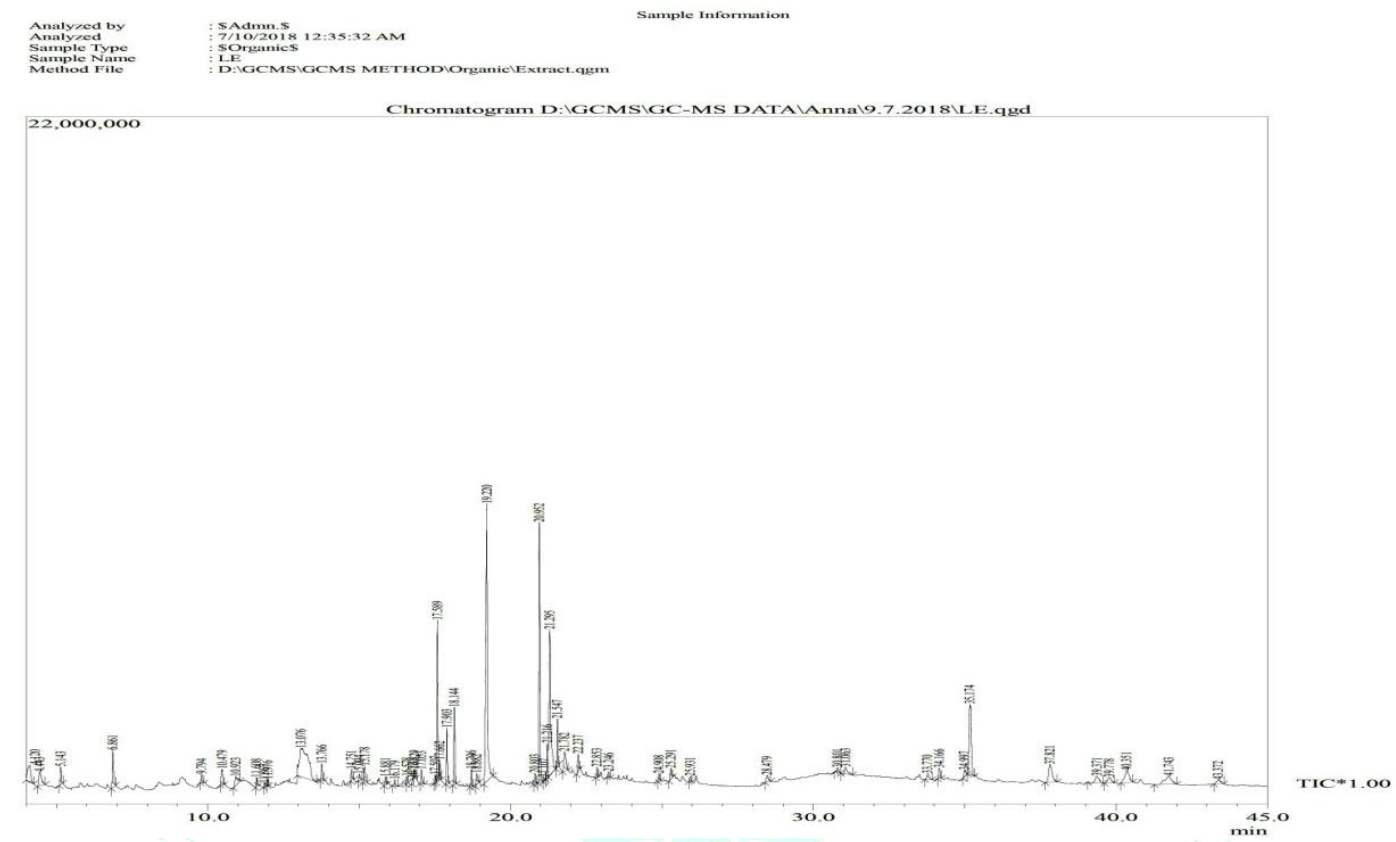

Figure 1: GC_MS Chromatogram of methanolic leaf extract of $L$. esculentum after SLF treatment

Table 1: Preliminary phytochemical analysis of leaf of SLF treated L. esculentum

\begin{tabular}{|l|c|c|c|c|}
\hline Phytochemicals & PE & CH & EA & E \\
\hline Alkaloids & - & - & + & + \\
\hline Carbohydrates & + & - & + & + \\
\hline Saponins & - & + & - & - \\
\hline Glycosides & - & - & - & + \\
\hline Amino acids & + & - & - & + \\
\hline Phytosterol & + & + & + & + \\
\hline Phenolic compounds & + & + & + & + \\
\hline Flavonoids & - & - & + & + \\
\hline Terpinoids & - & - & - & + \\
\hline Tannins & + & - & + & + \\
\hline
\end{tabular}

PE-Petroleum ether, CH-Chloroform, EA-Ethyl acetate, E-Ethanol, M-Methanol extract; (+) Positive, (-) Negative.

Table 2: FT-IR absorption and functional groups of leaf extract of $10 \%$ of SLF treated L. esculentum

\begin{tabular}{|l|l|l|l|l|}
\hline S. No & Wave No. & Molecular Motion & Functional group & Absorption Intensity \\
\hline 1 & 3417.43 & O-H stretch & Alcohol & Strong \\
\hline 2 & 2969.11 & O-H stretch & Alcohol & Medium \\
\hline 3 & 2950.60 & O-H stretch & Alcohol & Medium \\
\hline 4 & 2867.05 & C=O stretch & Aldehyde & Weak \\
\hline 5 & 2844.29 & C=O stretch & Aldehyde & Weak \\
\hline 6 & 2526.67 & C=N stretch & Iso cyanides & Medium \\
\hline 7 & 2076.59 & C=N stretch & Iso cyanides & Medium \\
\hline 8 & 2052.84 & C=N stretch & Iso cyanides & Strong \\
\hline 9 & 1647.40 & C=N stretch & Alkyl compound & Medium \\
\hline 10 & 1454.89 & C-H stretch & Alkane & Medium \\
\hline 11 & 1413.17 & C-H stretch & Alkane & Strong \\
\hline 12 & 1112.14 & C-O stretch & Primary alcohol & Strong \\
\hline 13 & 1054.59 & C-O stretch & Primary alcohol & Strong \\
\hline 14 & 1032.15 & C-O stretch & Primary alcohol & Strong \\
\hline 15 & 1015.72 & C-O stretch & Primary alcohol & Strong \\
\hline 16 & 658.20 & C-Cl stretch & Chloro compound & \\
\hline
\end{tabular}


Table 3: Phytoconstituents present in the Methanolic leaf extract of SLF treated L. esculentum using GC-MS

\begin{tabular}{|c|c|c|c|c|c|}
\hline Peak & R.Time & $\%$ of Area & $\begin{array}{l}\text { Molecular } \\
\text { formula }\end{array}$ & $\begin{array}{l}\text { Molecular } \\
\text { weight }\end{array}$ & Name of the compounds \\
\hline 1 & 4.120 & 2.13 & $\mathrm{C}_{3} \mathrm{H}_{8} \mathrm{O}_{3}$ & 92 & 1,2,3-PROPANETRIOL \\
\hline 2 & 4.443 & 1.52 & $\mathrm{C}_{8} \mathrm{H}_{17} \mathrm{NO}$ & 143 & Oxazolidine, 2,2-diethyl-3-methyl- \\
\hline 3 & 5.143 & 0.77 & $\mathrm{C}_{8} \mathrm{H}_{8} \mathrm{O}$ & 120 & Benzeneacetaldehyde \\
\hline 4 & 6.861 & 1.81 & $\mathrm{C}_{6} \mathrm{H}_{8} \mathrm{O}_{4}$ & 144 & 4H-Pyran-4-one, 2,3-dihydro-3,5-dihydroxy-6-methyl- \\
\hline 5 & 9.794 & 0.43 & $\mathrm{C}_{9} \mathrm{H}_{10} \mathrm{O}_{2}$ & 150 & 2-METHOXY-4-VINYLPHENOL \\
\hline 6 & 10.923 & 0.88 & $\mathrm{C}_{10} \mathrm{H}_{14} \mathrm{~N}_{20}$ & 178 & 3-(1-METHYL-2-PYRROLIDINYL)-,1-OXIDE \\
\hline 7 & 11.608 & 0.33 & $\mathrm{C}_{13} \mathrm{H}_{16} \mathrm{O}$ & 188 & Ethanone, 1-(2,3-dihydro-1,1-dimethyl-1H-inden-4-yl)- \\
\hline 8 & 11.867 & 0.09 & $\mathrm{C}_{4} \mathrm{H}_{4} \mathrm{O}$ & 68 & Furan \\
\hline 9 & 11.976 & 0.17 & $\mathrm{C}_{13} \mathrm{H}_{16} \mathrm{O}$ & 188 & 3,3-Dimethyl-4-phenyl-4-penten-2-one \\
\hline 10 & 13.076 & 10.19 & $\mathrm{C}_{6} \mathrm{H}_{12} \mathrm{O}_{6}$ & 180 & D-Allose \\
\hline 11 & 13.766 & 0.83 & $\mathrm{C}_{13} \mathrm{H}_{18} \mathrm{O}_{3}$ & 222 & 3-(tert-Butyl)-4-methoxyphenyl acetate \\
\hline 12 & 14.751 & 0.49 & $\mathrm{C}_{13} \mathrm{H}_{18} \mathrm{O}$ & 190 & MEGASTIGMATRIENONE 2 \\
\hline 13 & 15.178 & 0.88 & $\mathrm{C}_{14} \mathrm{H}_{22} \mathrm{O}$ & 206 & $\begin{array}{l}\text { Bicyclo[4.3.0]nonan-1-ol, 7,9-bis(methylene)-2,2,6- } \\
\text { trimethy }\end{array}$ \\
\hline 14 & 15.881 & 0.48 & $\mathrm{C}_{15} \mathrm{H}_{22} \mathrm{O}$ & 218 & 6-ISOPROPENYL-4,8A-DIMETHYL-3,5,6,7,8,8A-HEXAH \\
\hline 15 & 16.179 & 0.33 & $\mathrm{C}_{10} \mathrm{H}_{12} \mathrm{O}_{4}$ & 196 & Ethanone, 1-(4-hydroxy-3,5-dimethoxyphenyl)- \\
\hline 16 & 16.578 & 0.25 & $\mathrm{C}_{14} \mathrm{H}_{28} \mathrm{O}_{2}$ & 228 & Tetradecanoic acid \\
\hline 17 & 16.829 & 0.33 & $\mathrm{C}_{11} \mathrm{H}_{16} \mathrm{O}_{3}$ & 196 & $\begin{array}{l}\text { 6-Hydroxy-4,4,7a-trimethyl-5,6,7,7a- } \\
\text { tetrahydrobenzofuran-2 }\end{array}$ \\
\hline 18 & 17.507 & 0.27 & $\mathrm{C}_{20} \mathrm{H}_{40}$ & 280 & 2-Hexadecene, 3,7,11,15-tetramethyl-, [R-[R*,R*-(E)]]- \\
\hline 19 & 17.589 & 4.85 & $\mathrm{C}_{20} \mathrm{H}_{38}$ & 278 & Neophytadiene \\
\hline 20 & 18.144 & 2.55 & $\mathrm{C}_{22} \mathrm{H}_{42} \mathrm{O}_{2}$ & 338 & Phytol \\
\hline 21 & 18.706 & 0.56 & $\mathrm{C}_{16} \mathrm{H}_{30} \mathrm{O}_{4}$ & 270 & Hexadecanoic acid, methyl ester \\
\hline 22 & 18.882 & 0.65 & $\mathrm{C}_{17} \mathrm{H}_{28}$ & 232 & 1,8,11,14-Heptadecatetraene, $(\mathrm{Z}, \mathrm{Z}, \mathrm{Z})$ - \\
\hline 23 & 21.216 & 1.76 & $\mathrm{C}_{10 \mathrm{H}_{18}}$ & 138 & CYCLODECENE \\
\hline 24 & 21.547 & 1.70 & $\mathrm{C}_{18} \mathrm{H}_{36} \mathrm{O}_{2}$ & 284 & Octadecanoic acid \\
\hline 25 & 22.237 & 0.71 & $\mathrm{C}_{16} \mathrm{H}_{28} \mathrm{O}_{2}$ & 252 & OXACYCLOHEPTADEC-8-EN-2-ONE \\
\hline 26 & 24.908 & 0.31 & $\mathrm{C}_{12} \mathrm{H}_{23} \mathrm{NO}_{2}$ & 213 & 3-Cyclopentylpropionic acid, 2-dimethylaminoethyl ester \\
\hline 27 & 25.291 & 0.66 & $\mathrm{C}_{16} \mathrm{H}_{31} \mathrm{CIO}$ & 274 & Palmitoyl chloride \\
\hline 28 & 25.931 & 0.26 & $\mathrm{C}_{24} \mathrm{H}_{38} \mathrm{O}_{4}$ & 390 & Bis(2-ethylhexyl) phthalate \\
\hline 29 & 30.801 & 0.35 & $\mathrm{C}_{29} \mathrm{H}_{50} \mathrm{O}_{4}$ & 462 & .alpha.-Tocospiro B \\
\hline 30 & 31.063 & 0.54 & $\mathrm{C}_{28} \mathrm{H}_{44} \mathrm{~N}_{2} \mathrm{O}_{7}$ & 520 & .1-Pyrrolidinebutanoic acid \\
\hline 31 & 33.770 & 0.51 & $\mathrm{C}_{28} \mathrm{H}_{48} \mathrm{O}_{2}$ & 416 & gamma.-Tocopherol \\
\hline 32 & 34.166 & 0.67 & $\mathrm{C}_{31} \mathrm{H}_{50} \mathrm{O}_{3}$ & 470 & METHYL COMMATE B \\
\hline 33 & 34.997 & 0.47 & $\mathrm{C}_{27} \mathrm{H}_{46} \mathrm{O}$ & 386 & CHOLEST-5-EN-3-OL (3.BETA.)- \\
\hline 34 & 37.821 & 2.46 & $\mathrm{C}_{29} \mathrm{H}_{48} \mathrm{O}$ & 412 & Stigmasterol \\
\hline 35 & 39.371 & 0.87 & $\mathrm{C}_{29} \mathrm{H}_{50} \mathrm{O}$ & 414 & gamma.-Sitosterol \\
\hline 36 & 39.778 & 0.77 & $\mathrm{C}_{29} \mathrm{H}_{48} \mathrm{O}$ & 412 & Fucosterol \\
\hline 37 & 40.351 & 2.08 & $\mathrm{C}_{30} \mathrm{H}_{50} \mathrm{O}$ & 426 & beta.-Amyrin \\
\hline 38 & 41.743 & 2.20 & $\mathrm{C}_{34} \mathrm{H}_{54} \mathrm{O}_{3}$ & 426 & Lupeol myristate \\
\hline \multirow[t]{2}{*}{39} & 43.372 & 0.61 & $\mathrm{C}_{29} \mathrm{H}_{50} \mathrm{O}_{2}$ & 430 & Vitamin E \\
\hline & & 100.00 & & & \\
\hline
\end{tabular}

Table 4: Phytoconstituents present in the Methanolic leaf extract of untreated L. esculentum using GC-MS

\begin{tabular}{|c|c|c|c|c|c|}
\hline Peak & R.Time & $\begin{array}{l}\% \text { of } \\
\text { Area }\end{array}$ & $\begin{array}{l}\text { Molecular } \\
\text { formula }\end{array}$ & $\begin{array}{l}\text { Molecular } \\
\text { weight }\end{array}$ & Name of the compounds \\
\hline 1 & 4.432 & 0.88 & $\mathrm{C}_{14} \mathrm{H}_{22} \mathrm{O}$ & 206 & $\begin{array}{l}\text { Bicyclo[4.3.0]nonan-1-ol, 7,9-bis(methylene)-2,2,6- } \\
\text { trimethy }\end{array}$ \\
\hline 2 & 4.443 & 0.48 & $\mathrm{C}_{15} \mathrm{H}_{22} \mathrm{O}$ & 218 & 6-ISOPROPENYL-4,8A-DIMETHYL-3,5,6,7,8,8A-HEXAH \\
\hline 3 & 5.143 & 0.33 & $\mathrm{C}_{10} \mathrm{H}_{12} \mathrm{O}_{4}$ & 196 & Ethanone, 1-(4-hydroxy-3,5-dimethoxyphenyl)- \\
\hline 4 & 6.861 & 0.25 & $\mathrm{C}_{14} \mathrm{H}_{28} \mathrm{O}_{2}$ & 228 & Tetradecanoic acid \\
\hline 5 & 9.794 & 0.33 & $\mathrm{C}_{11} \mathrm{H}_{16} \mathrm{O}_{3}$ & 196 & $\begin{array}{l}\text { 6-Hydroxy-4,4,7a-trimethyl-5,6,7,7a- } \\
\text { tetrahydrobenzofuran-2 }\end{array}$ \\
\hline 6 & 10.923 & 0.27 & $\mathrm{C}_{20} \mathrm{H}_{40}$ & 280 & 2-Hexadecene, 3,7,11,15-tetramethyl-, [R-[R*, $\mathrm{R}^{*}$-(E)]]- \\
\hline 7 & 11.608 & 4.85 & $\mathrm{C}_{20} \mathrm{H}_{38}$ & 278 & Neophytadiene \\
\hline 8 & 11.867 & 2.55 & $\mathrm{C}_{22} \mathrm{H}_{42} \mathrm{O}_{2}$ & 338 & Phytol \\
\hline 9 & 11.976 & 0.56 & $\mathrm{C}_{16} \mathrm{H}_{30} \mathrm{O}_{4}$ & 270 & Hexadecanoic acid, methyl ester \\
\hline 10 & 11.608 & 0.65 & $\mathrm{C}_{17} \mathrm{H}_{28}$ & 232 & 1,8,11,14-Heptadecatetraene, (Z,Z,Z)- \\
\hline 11 & 11.867 & 1.76 & $\mathrm{C}_{10 H_{18}}$ & 138 & CYCLODECENE \\
\hline 12 & 11.976 & 1.70 & $\mathrm{C}_{18} \mathrm{H}_{36} \mathrm{O}_{2}$ & 284 & Octadecanoic acid \\
\hline 13 & 13.076 & 0.71 & $\mathrm{C}_{16} \mathrm{H}_{28} \mathrm{O}_{2}$ & 252 & OXACYCLOHEPTADEC-8-EN-2-ONE \\
\hline 14 & 14.085 & 0.31 & $\mathrm{C}_{12} \mathrm{H}_{23} \mathrm{NO}_{2}$ & 213 & 3-Cyclopentylpropionic acid, 2-dimethylaminoethyl \\
\hline
\end{tabular}




\begin{tabular}{|l|l|l|l|l|l|}
\hline & & & & & ester \\
\hline 15 & 16.00 & 1.81 & $\mathbf{C}_{\mathbf{6}} \mathbf{H}_{\mathbf{8}} \mathbf{O}_{\mathbf{4}}$ & 144 & 4H-Pyran-4-one, 2,3-dihydro-3,5-dihydroxy-6-methyl- \\
\hline 16 & 16.09 & 0.43 & $\mathbf{C}_{\mathbf{9}} \mathbf{H}_{\mathbf{1 0}} \mathbf{O}_{\mathbf{2}}$ & 150 & 2-METHOXY-4-VINYLPHENOL \\
\hline 17 & 17.03 & 0.88 & $\mathbf{C}_{\mathbf{1 0}} \mathbf{H}_{\mathbf{1 4}} \mathbf{N}_{\mathbf{2 0}}$ & 178 & 3-(1-METHYL-2-PYRROLIDINYL)-,1-OXIDE \\
\hline 18 & 19.08 & 0.33 & $\mathbf{C}_{\mathbf{1 3}} \mathbf{H}_{\mathbf{1 6}} \mathbf{0}$ & 188 & Ethanone, 1-(2,3-dihydro-1,1-dimethyl-1H-inden-4-yl)- \\
\hline 19 & 21.05 & 0.09 & $\mathbf{C}_{\mathbf{4}} \mathbf{H}_{\mathbf{4}} \mathbf{0}$ & 68 & Furan \\
\hline 20 & 13.076 & 0.17 & $\mathbf{C}_{\mathbf{1 3}} \mathbf{H}_{\mathbf{1 6}} \mathbf{0}$ & 188 & 3,3-Dimethyl-4-phenyl-4-penten-2-one \\
\hline 21 & 13.766 & 10.19 & $\mathbf{C}_{\mathbf{6}} \mathbf{H}_{\mathbf{1 2}} \mathbf{O}_{\mathbf{6}}$ & 180 & D-Allose \\
\hline 22 & 21.547 & 12.12 & $\mathbf{C}_{\mathbf{1 0}} \mathbf{H}_{\mathbf{1 0}} \mathbf{F}_{\mathbf{5}}$ & 253 & Silane dimethyl \\
\hline 23 & 22.237 & 13.23 & $\mathbf{C}_{\mathbf{4}} \mathbf{H}_{\mathbf{4}} \mathbf{O}_{\mathbf{4}}$ & 116 & Fumaric acid \\
\hline 24 & 24.908 & 13.08 & $\mathbf{C}_{\mathbf{2 8}} \mathbf{H}_{\mathbf{5 8}}$ & 394 & Octacosane \\
\hline 25 & 9.794 & 15.00 & $\mathbf{C}_{\mathbf{2 1}} \mathbf{H}_{\mathbf{4}}$ & 296 & Hencicosane \\
\hline 26 & 16.179 & 0.33 & $\mathbf{C}_{\mathbf{1 0}} \mathbf{H}_{\mathbf{1 2}} \mathbf{O}_{\mathbf{4}}$ & 196 & Ethanone, 1-(4-hydroxy-3,5-dimethoxyphenyl)- \\
\hline 27 & 16.578 & 0.25 & $\mathbf{C}_{\mathbf{1 4}} \mathbf{H}_{\mathbf{2 8}} \mathbf{O}_{\mathbf{2}}$ & 228 & Tetradecanoic acid \\
\hline & & & & & $\begin{array}{l}\text { 6-Hydroxy-4,4,7a-trimethyl-5,6,7,7a- } \\
\text { tetrahydrobenzofuran-2 }\end{array}$ \\
\hline
\end{tabular}

The phytochemical constituents present in the SLF treated L.esculentum leaf extract was contain 39 and phytochemical components according to GC-MS spectra (Fg-1). The retention time of these components are and, have been confirmed by spectra. The percentage of area of mass peak 18.706, 43.372, 41.743, 40.351, 31.063, 37.821 and 21.547 are inconsistent with molecular formula of $\mathrm{C} 30 \mathrm{H} 50 \mathrm{O}, \mathrm{C} 30 \mathrm{H}_{50 O}$, C32H56O, C17H34O2, $\mathrm{C} 22 \mathrm{H} 42 \mathrm{O}_{2}$ and C18H36O2 (Table 2). Interpretation of mass spectrum of GC-MS was conducted using the database of National Institute of Standards and Technology (NIST08s) and WILE8 and FAME having more patterns. The spectrum of unknown component was compared with the spectrum of known component was compared with the spectrum of known components stored in NIST08S, WILEY8 and FAME library. The name, molecular formula, molecular weight and structure of the component of the test material were determined. The Phyto-components are responsible for various pharmacological action like antimicrobial and antioxidant anti-inflammation, anti-cancer, Hepatoprotective, cytotoxicity, cyto-protective etc. (Table 5) Lycopersicum esculentum has medicinal value the presence of these major constituents.

Table 5: Biological activity of specific phyto-components identified in Lycopersicum esculentum treated with SLF by GC-MS

\begin{tabular}{|c|c|c|c|}
\hline S.No & $\begin{array}{l}\text { Name of the } \\
\text { compound }\end{array}$ & Structure of the compound & Biological activity \\
\hline 1 & Beta-amyrin & + & $\begin{array}{l}\text { Anti-tumor, Analgesic } \\
\text { Antibacterial, Anti-inflammatory } \\
\text { sedative, Fungicide }\end{array}$ \\
\hline 2 & $\begin{array}{l}\text { Gamma- } \\
\text { Sistosterol }\end{array}$ & & $\begin{array}{l}\text { Antimicrobial, Anti-inflammatory, } \\
\text { Anticacer, Diuretic, Antiasthma, } \\
\text { Haepatoprotective }\end{array}$ \\
\hline 3 & Lupeol Myristate & $\sum_{\text {amagnify }}$ & $\begin{array}{l}\text { Anticancer, Antioxidant, anti- } \\
\text { inflammatory and fungicide. }\end{array}$ \\
\hline 4 & Octa deconic acid & "० & $\begin{array}{l}\text { Antiviral, Anti-tumor, Anti- } \\
\text { inflammatory sedative, fungicide. }\end{array}$ \\
\hline 5 & Phytol & & $\begin{array}{l}\text { Pesticide, perfumery, Anti-feedent, } \\
\text { Haepato protective, anti- } \\
\text { inflammatory, Analgesic } \\
\text { Antibacterial activity. }\end{array}$ \\
\hline 6 & Hexadecanic acid & ${ }_{0 .}$ & Cardio protective \\
\hline 7 & Stigmasterol & & $\begin{array}{l}\text { Cyto-toxicity against human, anti- } \\
\text { microbial, anti-inflammatory, } \\
\text { anticancer, antiasthma. }\end{array}$ \\
\hline 8 & Vitamin E & & $\begin{array}{l}\text { Antioxidant and Cyto-protective } \\
\text { activites }\end{array}$ \\
\hline
\end{tabular}


L. esculentum revealed the different functional groups based on the FT-IR spectrum such as alcohol, phenols, alkanes, aldehydes, isocyanides, alkane, primary alcohols and chloro compounds (Table 2) which shows major peak at $3417.43,296.11,2950.60,2867.05,2844.29,2526.67$, $2076.59,2052.84,1647.40,1454.89,1413.17,1112.14$,
$1054.59,1032.15,1015.72$ and $658.20 \mathrm{~cm}^{-1}$ respectively (Fig-2). Therefore, comparison of chemical constituents and pharmacological activities of these phytochemical constituents is helpful to elucidate the mechanism of therapeutic effects and active components from $L$. esculentum plants.

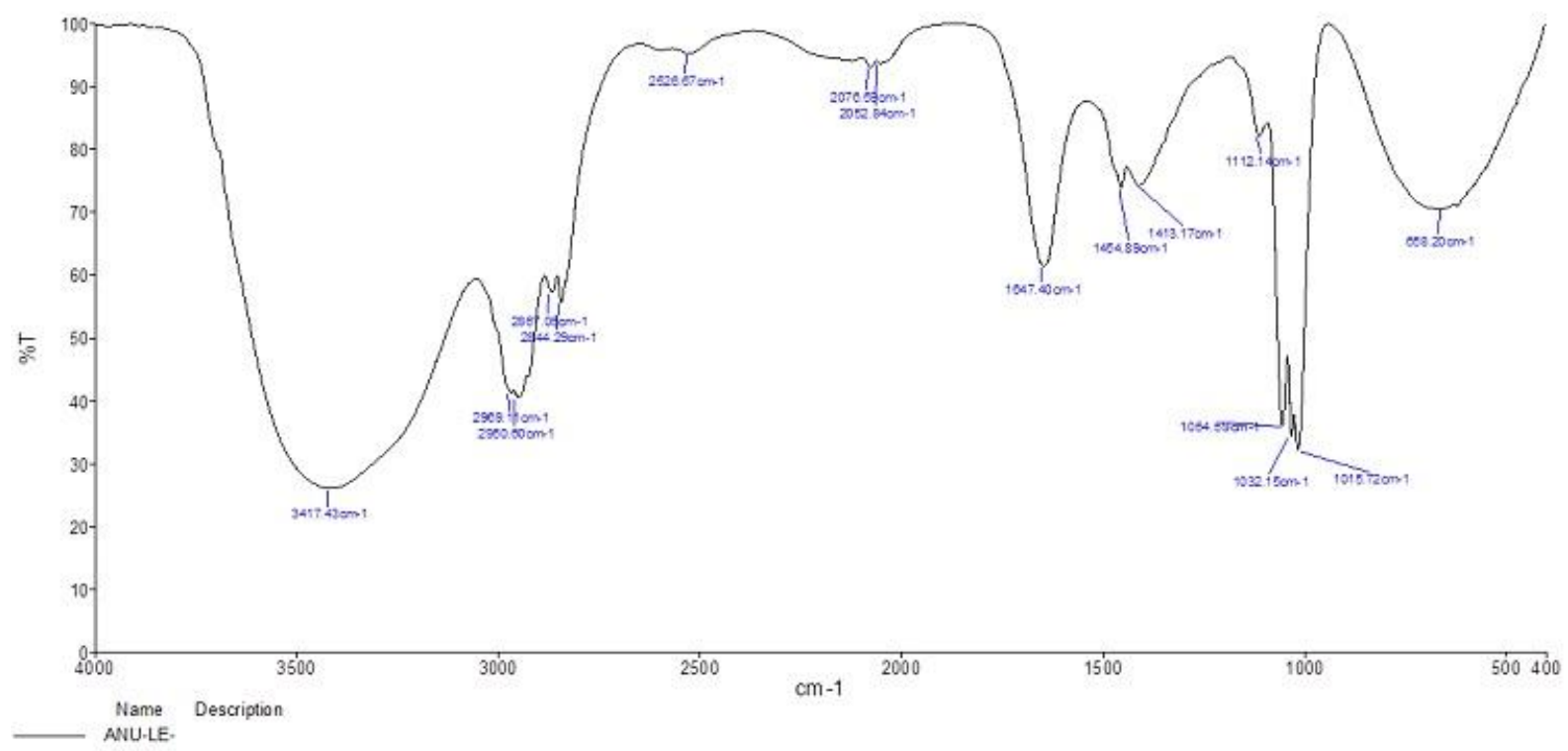

Figure 2: FT-IR Spectrum of methanolic leaf extract of L. esculentum

\section{CONCLUSION}

In conclusion about 39 phytochemical constituents were identified from the methanolic extract of L. esculentum after treatment with SLF. Since the identified phytochemical constituents biologically antimicrobial activity such as, antifungal and antiviral potential candidate possessing it is need to confirm their structure units sophisticated spectral frequencies in near future.

By using FT-IR spectrum we can conform the functional constituents from given extract, identify the medicinal material from the adulterate and even evaluate the quantities of medicinal materials. Many researches applied the FT-IR spectrum as tool for distinguish closely associated plants and other organisms. The results of the present study developed novel phytochemical marker to identify the medicinally important plant. Further advanced spectroscopic studies are required for the structural elucidation and identification of active principles present in the seaweed liquid fertilizer treated L. esculentum.

\section{REFERENCES}

1. Rajkumar S, Jebanesan A, Ovicidal activity of Solanum trilobatum Linn (Solanaceae); leaf extract against culex quinquefasciatus Say and Culex triaeniorhynchus Gile (Diptera: Culicidae: Intl. J. Trop. Sci, 2004; 24:340-342

2. Harbone JB, Phytochemical methods: Chapman and Hill (1973).

3. Vandana Mathur, Sharad Vats, Megha Jain, Jaya Bhojak and Raka Kamal. Antimicrobial Activity of Bioactive Metabolites Isolated from Selected Medicinal Plants: Asian J. Expt. Sci, 2007; 21:1-6.

4. Crouch IJ, Staden VJ, Evidence for the presence of plant growth regulators in commercial seaweed products: Plant Growth Regulat. 1993; 13:21-29.
The analyzed results of Gas Chromoatography-Mass spectroscopy of the methanol extract of tomato leaves ( $L$. esculentum) identified thirty nine compounds. The compounds identified may be an antimicrobial such as antifungal, antibacterial, antiviral and can be used to treat some diseases. In these results findings have shown that the SLF treated tomato leaf is extensively rich in secondary metabolites, compare with control leaf.

The present work delivered to establish the various phytochemical, GC-MS and FTIR parameters which could serve as important and has commercial interest in both research institute and pharmaceuticals companies for the manufacturing of innovation drugs. This primary information will facilitate in conducting further studies on discovery of bioactive molecules, resolve of their efficacy by in vivo studies and demonstration of their safety and efficacy in clinical trials. 